\title{
A Generalized Nonlinear Sum-Difference Inequality of Product Form
}

\author{
YongZhou Qin and Wu-Sheng Wang \\ School of Mathematics and Statistics, Hechi University, Guangxi, Yizhou 546300, China \\ Correspondence should be addressed to Wu-Sheng Wang; wang4896@126.com
}

Received 29 August 2013; Accepted 1 November 2013

Academic Editor: Mehmet Sezer

Copyright ( 2013 Y. Qin and W.-S. Wang. This is an open access article distributed under the Creative Commons Attribution License, which permits unrestricted use, distribution, and reproduction in any medium, provided the original work is properly cited.

\begin{abstract}
We establish a generalized nonlinear discrete inequality of product form, which includes both nonconstant terms outside the sums and composite functions of nonlinear function and unknown function without assumption of monotonicity. Upper bound estimations of unknown functions are given by technique of change of variable, amplification method, difference and summation, inverse function, and the dialectical relationship between constants and variables. Using our result we can solve both the discrete inequality in Pachpatte (1995). Our result can be used as tools in the study of difference equations of product form.
\end{abstract}

\section{Introduction}

Being an important tool in the study of existence, uniqueness, boundedness, stability, and other qualitative properties of solutions of differential equations and integral equations, various generalizations of Gronwall inequalities $[1,2]$ and their applications have attracted great interests of many mathematicians (such as [3-6]). Some recent works can be found, for example, in [7-10] and some references therein. Along with the development of the theory of integral inequalities and the theory of difference equations, more attention is paid to some discrete versions of Gronwall-Bellman type inequalities (such as $[3,4,11-13]$ ). Some recent works can be found, for example, in [14-24] and some references therein.

Pachpatte [4] obtained the explicit bound to the unknown function of the following sum-difference inequality:

$$
u^{2}(n) \leq\left(c_{1}^{2}+2 \sum_{s=0}^{n-1} f(s) u(s)\right)\left(c_{2}^{2}+2 \sum_{s=0}^{n-1} g(s) u(s)\right)
$$

Pachpatte [3] obtained the estimation of the unknown function of the following inequality:

$$
u(n) \leq\left(c_{1}+\sum_{s=0}^{n} f(s) u(s)\right)\left(c_{2}+\sum_{s=0}^{n} g(s) u(s)\right) .
$$

Then, the estimation can be used to study the boundedness, asymptotic behavior, and slow growth of the solutions of the sum-difference equation:

$$
\begin{aligned}
x(n)= & k\left(p_{1}(n)+\sum_{s=0}^{n-1} f_{1}(n-s) x(s)\right) \\
& \times\left(p_{2}(n)+\sum_{s=0}^{n-1} f_{2}(n-s) x(s)\right) .
\end{aligned}
$$

However, the bound given on such inequalities in $[3,4]$ is not directly applicable in the study of certain sum-difference equations. It is desirable to establish new inequalities of the above type, which can be used more effectively in the study of certain classes of sum-difference equations of product form.

In this paper, we establish a new integral inequality of product form

$$
\begin{aligned}
u(n) \leq & \left(p_{1}(n)+\sum_{s=0}^{n-1} f_{1}(n, s) \varphi_{1}(u(s))\right) \\
& \times\left(p_{2}(n)+\sum_{s=0}^{n-1} f_{2}(n, s) \varphi_{2}(u(s))\right), \quad \forall n \in \mathbb{N}_{0},
\end{aligned}
$$

where $p_{i}, f_{i}, \varphi_{i}(i=1,2)$ may not be monotone. For $\varphi_{1}$, $\varphi_{2}$, we employ a technique of monotonization to construct 
two functions; the second possesses stronger monotonicity than the first. We can demonstrate that inequalities (1) and (2), considered in $[3,4]$, respectively, can also be solved with our result. Finally, we expound that we can give estimation of solutions of a class of sum-difference equations of product form.

\section{Main Result and Proof}

In this section, we proceed to solve the discrete inequality (4) and present explicit bounds on the embedded unknown function. Let $\mathbb{N}_{0}:=\{0,1,2, \ldots\}, \mathbb{N}:=\{1,2, \ldots\}$, and $\mathbb{N}_{a}^{b}:=$ $\{a, a+1, a+2, \ldots, a+n=b\}\left(a \in \mathbb{N}_{0}, n, b \in \mathbb{N}\right)$. For function $z(n)$, its difference is defined by $\Delta z=z(n+$ 1) $-z(n)$. Obviously, the linear difference equation $\Delta z(n)=$ $b(n)$ with the initial condition $z\left(n_{0}\right)=0$ has the solution $z(n)=\sum_{s=n_{0}}^{n-1} b(s)$. For convenience, in the sequel we complementarily define that $\sum_{s=n_{0}}^{n_{0}-1} b(s)=0$.

First of all, we monotonize some given functions $p_{i}, f_{i}, \varphi_{i}$ in the sum; let

$$
q_{i}(n)=\max _{t \in \mathbb{N}_{0}^{n}} p_{i}(t), \quad g_{i}(n, s)=\max _{t \in \mathbb{N}_{0}^{n}} f_{i}(t, s), \quad i=1,2,
$$

where $q_{i}$ and $g_{i}$ are all nondecreasing in $n(i=1,2)$ and satisfy

$$
q_{i}(n) \geq p_{i}(n) \geq 0, \quad g_{i}(n, s) \geq f_{i}(n, s) \geq 0 .
$$

Let

$$
w_{1}(u)=\max _{s \in[0, u]} \varphi_{i}(s), \quad w_{2}(u):=\max _{s \in[0, u]}\left\{\frac{\varphi_{2}(s)}{w_{1}(s)}\right\} w_{1}(u),
$$

where $w_{i}(u)$ is nondecreasing in $u(i=1,2)$ and $w_{2}(u) / w_{1}(u)$ is also nondecreasing in $u$ and satisfies

$$
\begin{gathered}
w_{i}(u) \geq \varphi_{i}(u), \quad i=1,2, \\
W_{1}(x)=\int_{x_{0}}^{x} \frac{d s}{w_{1}(s)}, \quad x_{0}>0, \\
W_{2}(x):=\int_{x_{0}}^{x} \frac{w_{1}\left(W_{1}^{-1}(s)\right) d s}{w_{2}\left(W_{1}^{-1}(s)\right)}, \quad x_{0}>0, \\
W_{3}(x):=\int_{x_{0}}^{x} \frac{d s}{w_{1}\left(W_{1}^{-1}\left(W_{2}^{-1}(s)\right)\right)}, \quad x_{0}>0,
\end{gathered}
$$

where $W_{1}^{-1}, W_{2}^{-1}$ denote the inverse function of $W_{1}, W_{2}$, respectively.

Theorem 1. Let $f, g$ be nonnegative and given functions on $\mathbb{N}_{0}$. Suppose that $u$ is a nonnegative and unknown function. Then, the discrete inequality (4) gives

$$
u(n) \leq W_{1}^{-1}\left(W_{2}^{-1}\left(W_{3}^{-1}(A(n)+B(n))\right)\right), \quad \forall n \in \mathbb{N}_{0}^{b}
$$

where $W_{1}, W_{2}, W_{3}$ are defined by (9), (10), and (11), respectively, $W_{1}^{-1}, W_{2}^{-1}, W_{3}^{-1}$ denote the inverse functions of $W_{1}, W_{2}, W_{3}$, respectively,

$$
\begin{aligned}
& A(n)= W_{3}\left(W_{2}\left(W_{1}\left(q_{1}(n) q_{2}(n)\right)+\sum_{t=0}^{n-1} q_{2}(n) g_{1}(n, t)\right)\right. \\
&\left.\quad+\sum_{s=0}^{n-1} q_{1}(n) g_{2}(n, t)\right), \\
& B(n)=\sum_{t=0}^{n-1}\left(g_{2}(n, t) \sum_{s=0}^{t} g_{1}(n, s)+g_{1}(n, t) \sum_{s=0}^{t-1} g_{2}(n, s)\right)
\end{aligned}
$$

and $b$ is the largest natural number such that

$$
\begin{aligned}
b=\operatorname{Max}\{n & \in \mathbb{N}_{0}: A(n)+B(n) \in \operatorname{Dom}\left(W_{3}^{-1}\right), \\
& W_{3}^{-1}(A(n)+B(n)) \in \operatorname{Dom}\left(W_{2}^{-1}\right), \\
& \left.W_{2}^{-1}\left(W_{3}^{-1}(A(n)+B(n))\right) \in \operatorname{Dom}\left(W_{1}^{-1}\right)\right\} .
\end{aligned}
$$

Proof. Using (5), (6), (7), and (8), we observe that

$$
\begin{aligned}
u(n) \leq & \left(p_{1}(n)+\sum_{s=0}^{n-1} f_{1}(n, s) \varphi_{1}(u(s))\right) \\
& \times\left(p_{2}(n)+\sum_{s=0}^{n-1} f_{2}(n, s) \varphi_{2}(u(s))\right) \\
\leq & \left(q_{1}(n)+\sum_{s=0}^{n-1} g_{1}(n, s) w_{1}(u(s))\right) \\
& \times\left(q_{2}(n)+\sum_{s=0}^{n-1} g_{2}(n, s) w_{2}(u(s))\right) \\
\leq & \left(q_{1}(T)+\sum_{s=0}^{n-1} g_{1}(T, s) w_{1}(u(s))\right) \\
& \times\left(q_{2}(T)+\sum_{s=0}^{n-1} g_{2}(T, s) w_{2}(u(s))\right), \quad \forall n \in \mathbb{N}_{0}^{T},
\end{aligned}
$$

where $T \in \mathbb{N}_{0}^{J}$ is chosen arbitrarily. Let $v(n)$ denote the function on the right-hand side of (15), namely,

$$
\begin{aligned}
v(n)= & \left(q_{1}(T)+\sum_{s=0}^{n-1} g_{1}(T, s) w_{1}(u(s))\right) \\
& \times\left(q_{2}(T)+\sum_{s=0}^{n-1} g_{2}(T, s) w_{2}(u(s))\right), \quad \forall n \in \mathbb{N}_{0}^{T},
\end{aligned}
$$

which is a nonnegative and nondecreasing function on $\mathbb{N}_{0}^{T}$ with $v(0)=q_{1}(T) q_{2}(T)$. Then (4) is equivalent to

$$
u(n) \leq v(n), \quad \forall n \in \mathbb{N}_{0}^{T} .
$$


Using the difference formula

$$
\Delta x(n) y(n)=x(n+1) \Delta y(n)+y(n) \Delta x(n)
$$

and the monotonicity of $w_{i}$ and $v$, from (16) and (17), we observe that

$$
\begin{aligned}
\Delta v(n)= & \left(q_{1}(T)+\sum_{s=0}^{n-1} g_{1}(T, s) w_{1}(u(s))\right) \\
& \times \Delta\left(q_{2}(T)+\sum_{s=0}^{n-1} g_{2}(T, s) w_{2}(u(s))\right) \\
& +\Delta\left(q_{1}(T)+\sum_{s=0}^{n-1} g_{1}(T, s) w_{1}(u(s))\right) \\
& \times\left(q_{2}(T)+\sum_{s=0}^{n-1} g_{2}(T, s) w_{2}(u(s))\right) \\
= & \left.q_{1}(T)+\sum_{s=0}^{n} g_{1}(T, s) w_{1}(u(s))\right) \\
& \times g_{2}(T, n) w_{2}(u(n)) \\
& +g_{1}(T, n) w_{1}(u(n)) \\
& \times g_{1}(T, n) w_{1}(v(n)) \\
& \times\left(q_{2}(T)+\sum_{s=0}^{n} g_{1}(T, s) w_{2}(v)+\sum_{s=0}(v(s))\right) \\
& \left.(T, s) w_{2}(u(s))\right)
\end{aligned}
$$

$$
\begin{aligned}
& +q_{1}(T) g_{2}(T, n) \frac{w_{2}(v(n))}{w_{1}(v(n))} \\
& +g_{2}(T, n) \frac{w_{2}(v(n))}{w_{1}(v(n))} \\
& \times \sum_{s=0}^{n} g_{1}(T, s) w_{1}(v(s)) \\
& \left.+g_{1}(T, n) \sum_{s=0}^{n-1} g_{2}(T, s) w_{2}(v(s))\right)
\end{aligned}
$$

$$
\leq w_{1}(v(n))\left(q_{2}(T) g_{1}(T, n)\right.
$$

$$
+q_{1}(T) g_{2}(T, n) \frac{w_{2}(v(n))}{w_{1}(v(n))}
$$$$
+\left(g_{2}(T, n) \sum_{s=0}^{n} g_{1}(T, s)\right.
$$

$$
\left.+g_{1}(T, n) \sum_{s=0}^{n-1} g_{2}(T, s)\right)
$$$$
\left.\times w_{2}(v(n))\right)
$$

$$
\forall n \in \mathbb{N}_{0}^{T}
$$

for all $n \in \mathbb{N}_{0}^{T}$. From (19), we have

$$
\begin{aligned}
\frac{\Delta v(n)}{w_{1}(v(n))} \leq & q_{2}(T) g_{1}(T, n)+q_{1}(T) g_{2}(T, n) \frac{w_{2}(v(n))}{w_{1}(v(n))} \\
& +\left(g_{2}(T, n) \sum_{s=0}^{n} g_{1}(T, s)+g_{1}(T, n)\right. \\
& \left.\times \sum_{s=0}^{n-1} g_{2}(T, s)\right) w_{2}(v(n)), \quad \forall n \in \mathbb{N}_{0}^{T} .
\end{aligned}
$$

On the other hand, by the mean value theorem for integrals, for arbitrarily given integers $n, n+1 \in \mathbb{N}_{0}^{T}$, there exists $\xi$ in the open interval $(v(n), v(n+1))$ such that

$$
\begin{aligned}
W_{1}(v(n+1))-W_{1}(v(n)) \\
\quad=\int_{v(n)}^{v(n+1)} \frac{d s}{w_{1}(s)}=\frac{\Delta v(n)}{w_{1}(\xi)} \leq \frac{\Delta v(n)}{w_{1}(v(n))}, \quad \forall n \in \mathbb{N}_{0}^{T},
\end{aligned}
$$

where $W_{1}$ is defined by (9). From (20) and (21), we have

$$
\begin{aligned}
& W_{1}(v(n+1))-W_{1}(v(n)) \\
& \leq q_{2}(T) g_{1}(T, n)+q_{1}(T) g_{2}(T, n) \frac{w_{2}(v(n))}{w_{1}(v(n))} \\
& +\left(g_{2}(T, n) \sum_{s=0}^{n} g_{1}(T, s)\right. \\
& \left.\quad+g_{1}(T, n) \sum_{s=0}^{n-1} g_{2}(T, s)\right) w_{2}(v(n)),
\end{aligned}
$$


for all $n \in \mathbb{N}_{0}^{T}$. By setting $n=t$ in (22) and substituting $t=$ $0,1,2, \ldots, n-1$ successively, we obtain

$$
\begin{aligned}
W_{1}(v(n)) \leq & W_{1}(v(0)) \\
& +\sum_{t=0}^{n-1} q_{2}(T) g_{1}(T, t) \\
& +\sum_{t=0}^{n-1} q_{1}(T) g_{2}(T, t) \frac{w_{2}(v(t))}{w_{1}(v(t))} \\
& +\sum_{t=0}^{n-1}\left(g_{2}(T, t) \sum_{s=0}^{t} g_{1}(T, s)\right. \\
& \left.+W_{1}(v(0))+\sum_{t=0}^{T-1} q_{2}(T) \sum_{s=0}^{t-1} g_{2}(T, s)\right) w_{2}(v(t)) \\
& +\sum_{t=0}^{n-1} q_{1}(T) g_{2}(T, t) \frac{w_{2}(v(t))}{w_{1}(v(t))} \\
& +\sum_{t=0}^{n-1}\left(g_{2}(T, t) \sum_{s=0}^{t} g_{1}(T, s)\right. \\
& \left.+g_{1}(T, t) \sum_{s=0}^{t-1} g_{2}(T, s)\right) w_{2}(v(t)),
\end{aligned}
$$

Let $x(n)$ denote the function on the right-hand side of (23); namely,

$$
\begin{aligned}
& x(n)= W_{1}(v(0))+\sum_{t=0}^{T-1} q_{2}(T) g_{1}(T, t) \\
&+\sum_{t=0}^{n-1} q_{1}(T) g_{2}(T, t) \frac{w_{2}(v(t))}{w_{1}(v(t))} \\
&+\sum_{t=0}^{n-1}\left(g_{2}(T, t) \sum_{s=0}^{t} g_{1}(T, s)\right. \\
&\left.+g_{1}(T, t) \sum_{s=0}^{t-1} g_{2}(T, s)\right) w_{2}(v(t)), \\
& \forall n \in \mathbb{N}_{0}^{T} .
\end{aligned}
$$

Then $x(0)=W_{1}(v(0))+\sum_{t=0}^{T-1} q_{2}(T) g_{1}(T, t), x$ is a nonnegative and nondecreasing function on $\mathbb{N}_{0}^{T}$, and (23) is equivalent to

$$
v(n) \leq W_{1}^{-1}(x(n)), \quad \forall n \in \mathbb{N}_{0}^{T} .
$$

From (24), we obtain

$$
\begin{aligned}
\Delta x(n)= & q_{1}(T) g_{2}(T, n) \frac{w_{2}(v(n))}{w_{1}(v(n))} \\
& +\left(g_{2}(T, n) \sum_{s=0}^{n} g_{1}(T, s)\right. \\
& \left.+g_{1}(T, n) \sum_{s=0}^{n-1} g_{2}(T, s)\right) w_{2}(v(n)) \\
\leq & q_{1}(T) g_{2}(T, n) \frac{w_{2}\left(W_{1}^{-1}(x(n))\right)}{w_{1}\left(W_{1}^{-1}(x(n))\right)} \\
& +\left(g_{2}(T, n) \sum_{s=0}^{n} g_{1}(T, s)\right. \\
& \left.+g_{1}(T, n) \sum_{s=0}^{n-1} g_{2}(T, s)\right) w_{2}\left(W_{1}^{-1}(x(n))\right)
\end{aligned}
$$

From (26), we have

$$
\begin{aligned}
& \frac{w_{1}\left(W_{1}^{-1}(x(n))\right) \Delta x(n)}{w_{2}\left(W_{1}^{-1}(x(n))\right)} \\
& \leq q_{1}(T) g_{2}(T, n) \\
& \quad+\left(g_{2}(T, n) \sum_{s=0}^{n} g_{1}(T, s)\right. \\
& \left.\quad+g_{1}(T, n) \sum_{s=0}^{n-1} g_{2}(T, s)\right) w_{1}\left(W_{1}^{-1}(x(n))\right), \\
& \quad \forall n \in \mathbb{N}_{0}^{T} .
\end{aligned}
$$

Once again, performing the same procedure as in (21), (22), and (23), (27) gives

$$
\begin{gathered}
W_{2}(x(n)) \leq W_{2}(x(0))+\sum_{s=0}^{n-1} q_{1}(T) g_{2}(T, t) \\
+\sum_{s=0}^{n-1}\left(g_{2}(T, t) \sum_{s=0}^{t} g_{1}(T, s)\right. \\
\left.+g_{1}(T, t) \sum_{s=0}^{t-1} g_{2}(T, s)\right) \\
\times w_{1}\left(W_{1}^{-1}(x(t))\right)
\end{gathered}
$$




$$
\begin{gathered}
\leq W_{2}(x(0))+\sum_{s=0}^{T-1} g_{1}(T) g_{2}(T, t) \\
+\sum_{s=0}^{n-1}\left(g_{2}(T, t) \sum_{s=0}^{t} g_{1}(T, s)\right. \\
\left.+g_{1}(T, t) \sum_{s=0}^{t-1} g_{2}(T, s)\right) \\
\times w_{1}\left(W_{1}^{-1}(x(t))\right), \quad \forall n \in \mathbb{N}_{0}^{T},
\end{gathered}
$$

where $W_{2}$ is defined by (10). Let $z(n)$ denote the function on the right-hand side of (28); namely,

$$
\begin{array}{r}
z(n)=W_{2}(x(0))+\sum_{s=0}^{T-1} q_{1}(T) g_{2}(T, t) \\
+\sum_{s=0}^{n-1}\left(g_{2}(T, t) \sum_{s=0}^{t} g_{1}(T, s)\right. \\
\left.+g_{1}(T, t) \sum_{s=0}^{t-1} g_{2}(T, s)\right) w_{1}\left(W_{1}^{-1}(x(t))\right), \\
\forall n \in \mathbb{N}_{0}^{T} .
\end{array}
$$

Then $z(0)=W_{2}(x(0))+\sum_{s=0}^{T-1} q_{1}(T) g_{2}(T, t), z$ is a nonnegative and nondecreasing function on $\mathbb{N}_{0}^{T}$, and (28) is equivalent to

$$
x(n) \leq W_{2}^{-1}(z(n)), \quad \forall n \in \mathbb{N}_{0}^{T} .
$$

From (29) and (30), we obtain

$$
\begin{aligned}
\Delta z(n)= & \left(g_{2}(T, n) \sum_{s=0}^{n} g_{1}(T, s)\right. \\
& \left.\quad+g_{1}(T, n) \sum_{s=0}^{n-1} g_{2}(T, s)\right) w_{1}\left(W_{1}^{-1}(x(n))\right) \\
\leq & \left(g_{2}(T, n) \sum_{s=0}^{n} g_{1}(T, s)\right. \\
& \left.\quad+g_{1}(T, n) \sum_{s=0}^{n-1} g_{2}(T, s)\right) w_{1}\left(W_{1}^{-1}\left(W_{2}^{-1}(z(n))\right)\right),
\end{aligned}
$$

for all $n \in \mathbb{N}_{0}^{T}$. From (31), we have

$$
\begin{array}{r}
\frac{\Delta z(n)}{w_{1}\left(W_{1}^{-1}\left(W_{2}^{-1}(z(n))\right)\right)} \leq \\
g_{2}(T, n) \sum_{s=0}^{n} g_{1}(T, s) \\
+g_{1}(T, n) \sum_{s=0}^{n-1} g_{2}(T, s), \\
\forall n \in \mathbb{N}_{0}^{T} .
\end{array}
$$

Once again, performing the same procedure as in (21), (22), and (23), (32) gives

$$
\begin{aligned}
W_{3}(z(n)) \leq & W_{3}(z(0)) \\
+\sum_{t=0}^{n-1}( & g_{2}(T, t) \sum_{s=0}^{t} g_{1}(T, s) \\
& \left.\quad+g_{1}(T, t) \sum_{s=0}^{t-1} g_{2}(T, s)\right), \quad \forall n \in \mathbb{N}_{0}^{T} .
\end{aligned}
$$

Using (17), (25), and (30), from (33) we have

$u(n)$

$$
\begin{aligned}
\leq v(n) \leq W_{1}^{-1}(x(n)) \leq & W_{1}^{-1}\left(W_{2}^{-1}(z(n))\right) \\
\leq W_{1}^{-1}\left(W _ { 2 } ^ { - 1 } \left(W _ { 3 } ^ { - 1 } \left(W_{3}(z(0))\right.\right.\right. & \\
& +\sum_{t=0}^{n-1}\left(g_{2}(T, t) \sum_{s=0}^{t} g_{1}(T, s)\right. \\
& \left.\left.\left.\left.+g_{1}(T, t) \sum_{s=0}^{t-1} g_{2}(T, s)\right)\right)\right)\right)
\end{aligned}
$$$$
\leq W_{1}^{-1}\left(W _ { 2 } ^ { - 1 } \left(W _ { 3 } ^ { - 1 } \left(W _ { 3 } \left(W_{2}(x(0))\right.\right.\right.\right.
$$

$$
\left.+\sum_{s=0}^{T-1} q_{1}(T) g_{2}(T, t)\right)
$$$$
+\sum_{t=0}^{n-1}\left(g_{2}(T, t) \sum_{s=0}^{t} g_{1}(T, s)\right.
$$$$
\left.\left.\left.+g_{1}(T, t) \sum_{s=0}^{t-1} g_{2}(T, s)\right)\right)\right)
$$$$
\leq W_{1}^{-1}\left(W _ { 2 } ^ { - 1 } \left(W _ { 3 } ^ { - 1 } \left(W _ { 3 } \left(W _ { 2 } \left(W_{1}\left(q_{1}(T) q_{2}(T)\right)\right.\right.\right.\right.\right.
$$

$$
\left.+\sum_{t=0}^{T-1} q_{2}(T) g_{1}(T, t)\right)
$$

$$
\left.+\sum_{s=0}^{T-1} q_{1}(T) g_{2}(T, t)\right)
$$

$$
\begin{aligned}
+\sum_{t=0}^{n-1}\left(g_{2}(T, t) \sum_{s=0}^{t} g_{1}(T, s)\right. \\
\left.\left.\left.\left.+g_{1}(T, t) \sum_{s=0}^{t-1} g_{2}(T, s)\right)\right)\right)\right),
\end{aligned}
$$


As $n=T$, (34) yields

$u(T)$

$$
\begin{array}{r}
\leq W_{1}^{-1}\left(W _ { 2 } ^ { - 1 } \left(W _ { 3 } ^ { - 1 } \left(W _ { 3 } \left(W _ { 2 } \left(W_{1}\left(q_{1}(T) q_{2}(T)\right)\right.\right.\right.\right.\right. \\
\left.+\sum_{t=0}^{T-1} q_{2}(T) g_{1}(T, t)\right) \\
\left.+\sum_{s=0}^{T-1} q_{1}(T) g_{2}(T, t)\right) \\
+\sum_{t=0}^{T-1}\left(g_{2}(T, t) \sum_{s=0}^{t} g_{1}(T, s)\right. \\
\left.\left.\left.\left.+g_{1}(T, t) \sum_{s=0}^{t-1} g_{2}(T, s)\right)\right)\right)\right) .
\end{array}
$$

Since $T \in \mathbb{N}$, and $T \leq b$ is chosen arbitrarily in (35), the estimation (12) is derived. This completes the proof of Theorem 1.

\section{Application}

We consider a sum-difference equation of product form

$$
\begin{array}{r}
x(n)=\left(a(n)+\sum_{s=0}^{n-1} f(n, s) \varphi_{1}(x(s))\right) \\
\times\left(b(n)+\sum_{s=0}^{n-1} g(n, s) \varphi_{2}(x(s))\right), \\
\forall n \in \mathbb{N}_{0} .
\end{array}
$$

From (36), we have

$$
\begin{array}{r}
|x(n)| \leq\left(a(n)+\sum_{s=0}^{n-1} f(n, s) \varphi_{1}(|x(s)|)\right) \\
\times\left(b(n)+\sum_{s=0}^{n-1} g(n, s) \varphi_{2}(|x(s)|)\right), \\
\forall n \in \mathbb{N}_{0} .
\end{array}
$$

Let $u(n)=|x(n)|, p_{1}(n)=|a(n)|, p_{2}(n)=|b(n)|, f_{1}(n, s)=$ $|f(n, s)|$, and $f_{2}(n, s)=|g(n, s)|$ in (37); then (37) is the inequality of the form (4). Applying our result we get the estimation of solution of the sum-difference equations of product form (36).

\section{Acknowledgments}

This research was supported by National Natural Science Foundation of China (Project no. 11161018) and Guangxi Natural Science Foundation (2012GXNSFAA053009).

\section{References}

[1] T. H. Gronwall, "Note on the derivatives with respect to a parameter of the solutions of a system of differential equations," Annals of Mathematics, vol. 20, no. 4, pp. 292-296, 1919.

[2] R. Bellman, "The stability of solutions of linear differential equations," Duke Mathematical Journal, vol. 10, pp. 643-647, 1943.

[3] B. G. Pachpatte, "On a new inequality suggested by the study of certain epidemic models," Journal of Mathematical Analysis and Applications, vol. 195, no. 3, pp. 638-644, 1995.

[4] B. G. Pachpatte, "On some new inequalities related to certain inequalities in the theory of differential equations," Journal of Mathematical Analysis and Applications, vol. 189, no. 1, pp. 128144, 1995.

[5] R. P. Agarwal, S. Deng, and W. Zhang, "Generalization of a retarded Gronwall-like inequality and its applications," Applied Mathematics and Computation, vol. 165, no. 3, pp. 599-612, 2005.

[6] W.-S. Cheung, "Some new nonlinear inequalities and applications to boundary value problems," Nonlinear Analysis: Theory, Methods \& Applications, vol. 64, no. 9, pp. 2112-2128, 2006.

[7] W.-S. Wang, "A generalized retarded Gronwall-like inequality in two variables and applications to BVP," Applied Mathematics and Computation, vol. 191, no. 1, pp. 144-154, 2007.

[8] R. P. Agarwal, C. S. Ryoo, and Y.-H. Kim, "New integral inequalities for iterated integrals with applications," Journal of Inequalities and Applications, vol. 2007, Article ID 24385, 18 pages, 2007.

[9] A. Abdeldaim and M. Yakout, "On some new integral inequalities of Gronwall-Bellman-Pachpatte type," Applied Mathematics and Computation, vol. 217, no. 20, pp. 7887-7899, 2011.

[10] W. S. Wang, D. Huang, and X. Li, "Generalized retarded nonlinear integral inequalities involving iterated integrals and an application," Journal of Inequalities and Applications, vol. 2013, article 376, 2013.

[11] T. E. Hull and W. A. J. Luxemburg, "Numerical methods and existence theorems for ordinary differential equations," Numerische Mathematik, vol. 2, no. 1, pp. 30-41, 1960.

[12] D. Willett and J. S. W. Wong, "On the discrete analogues of some generalizations of Gronwall's inequality," vol. 69, pp. 362-367, 1965.

[13] B. G. Pachpatte and S. G. Deo, "Stability of discrete-time systems with retarded argument," Utilitas Mathematica, vol. 4, pp. 15-33, 1973.

[14] E. Yang, "A new nonlinear discrete inequality and its application," Annals of Differential Equations, vol. 17, no. 3, pp. 261-267, 2001.

[15] B. G. Pachpatte, "On some fundamental integral inequalities and their discrete analogues," Journal of Inequalities in Pure and Applied Mathematics, vol. 2, no. 2, article 15, 13 pages, 2001.

[16] F. W. Meng and W. N. Li, "On some new nonlinear discrete inequalities and their applications," Journal of Computational and Applied Mathematics, vol. 158, no. 2, pp. 407-417, 2003.

[17] W.-S. Cheung and J. Ren, "Discrete non-linear inequalities and applications to boundary value problems," Journal of Mathematical Analysis and Applications, vol. 319, no. 2, pp. 708-724, 2006.

[18] B. G. Pachpatte, Integral and Finite Difference Inequalities and Applications, vol. 205, Elsevier Science, Amsterdam, The Netherlands, 2006. 
[19] W. Sheng and W. N. Li, "Bounds on certain nonlinear discrete inequalities," Journal of Mathematical Inequalities, vol. 2, no. 2, pp. 279-286, 2008.

[20] Q.-H. Ma and W.-S. Cheung, "Some new nonlinear difference inequalities and their applications," Journal of Computational and Applied Mathematics, vol. 202, no. 2, pp. 339-351, 2007.

[21] W. S. Wang, "A generalized sum-difference inequality and applications to partial Difference equations," Advances in Difference Equations, vol. 2008, article 12, 2008.

[22] W. S. Wang, "Estimation on certain nonlinear discrete inequality and applications to boundary value problem," Advances in Difference Equations, vol. 2009, Article ID 708587, 2009.

[23] W. S. Wang, Z. Li, and W. S. Cheung, "Some new nonlinear retarded sum-difference inequalities with applications," Advances in Difference Equations, vol. 2011, article 41, 2011.

[24] H. Zhou, D. Huang, and W. S. Wang, "Some new difference inequalities and an application to discrete-time control systems," Journal of Applied Mathematics, vol. 2012, Article ID 214609, 14 pages, 2012. 


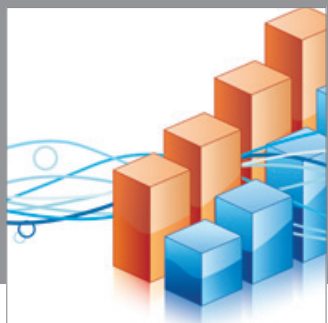

Advances in

Operations Research

mansans

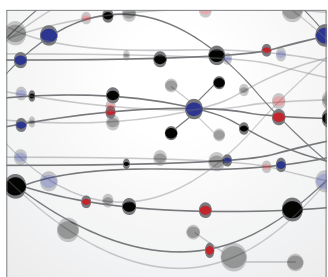

The Scientific World Journal
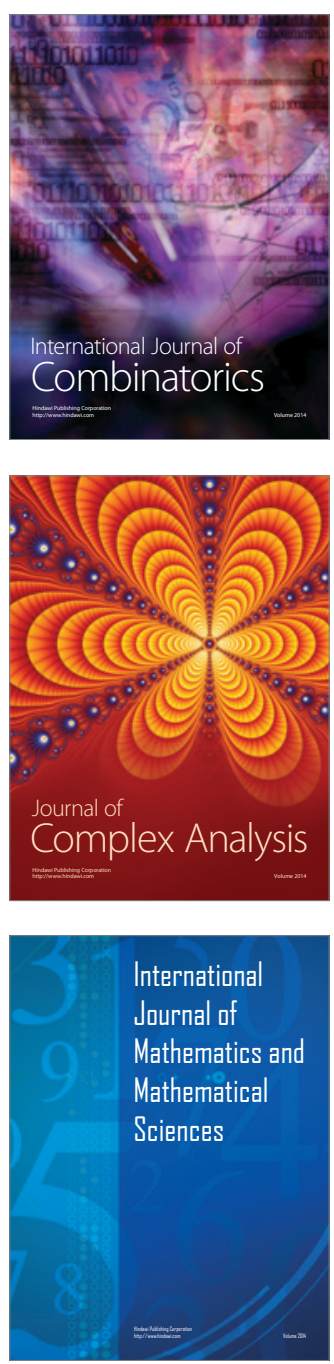
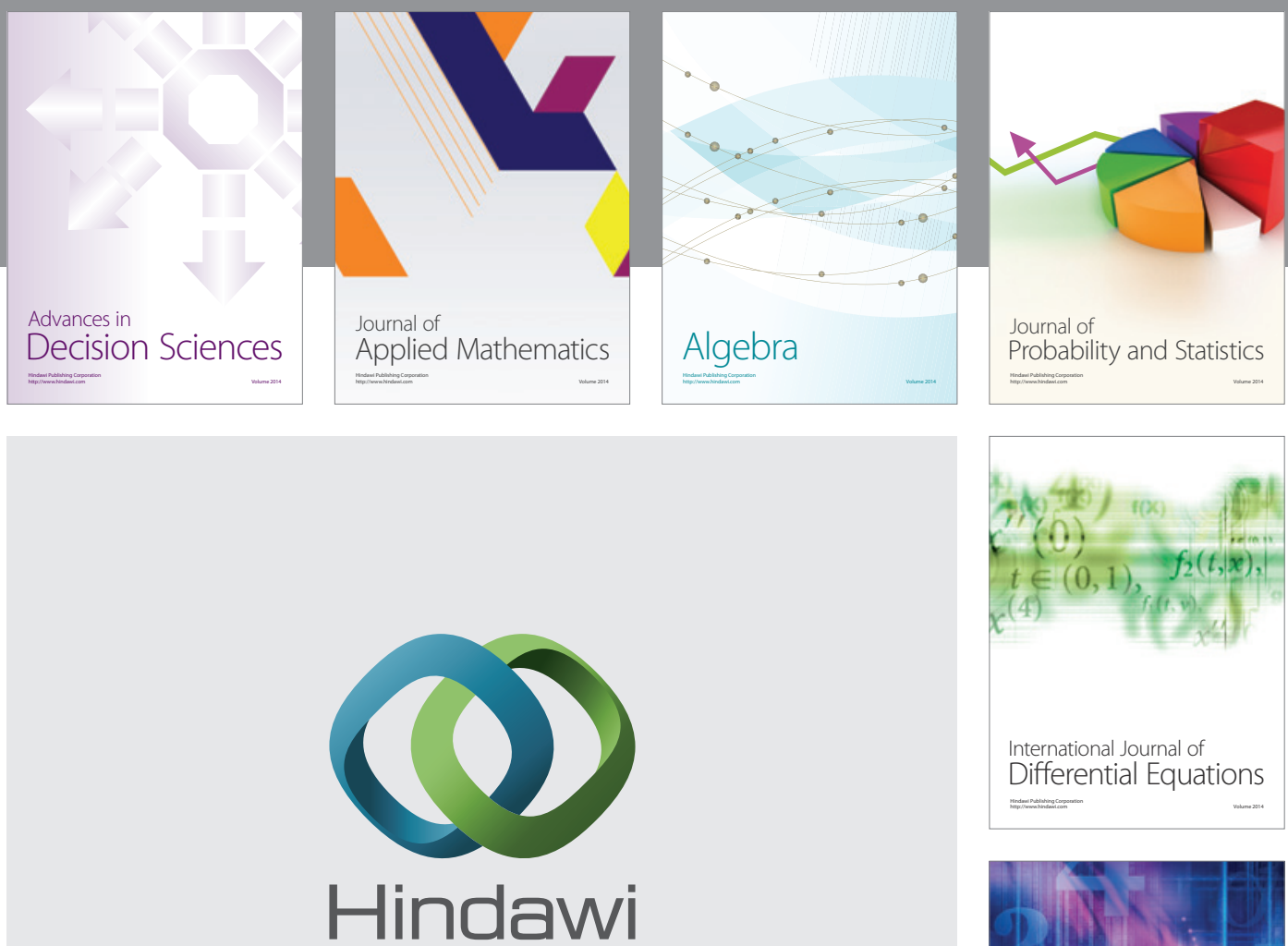

Submit your manuscripts at http://www.hindawi.com
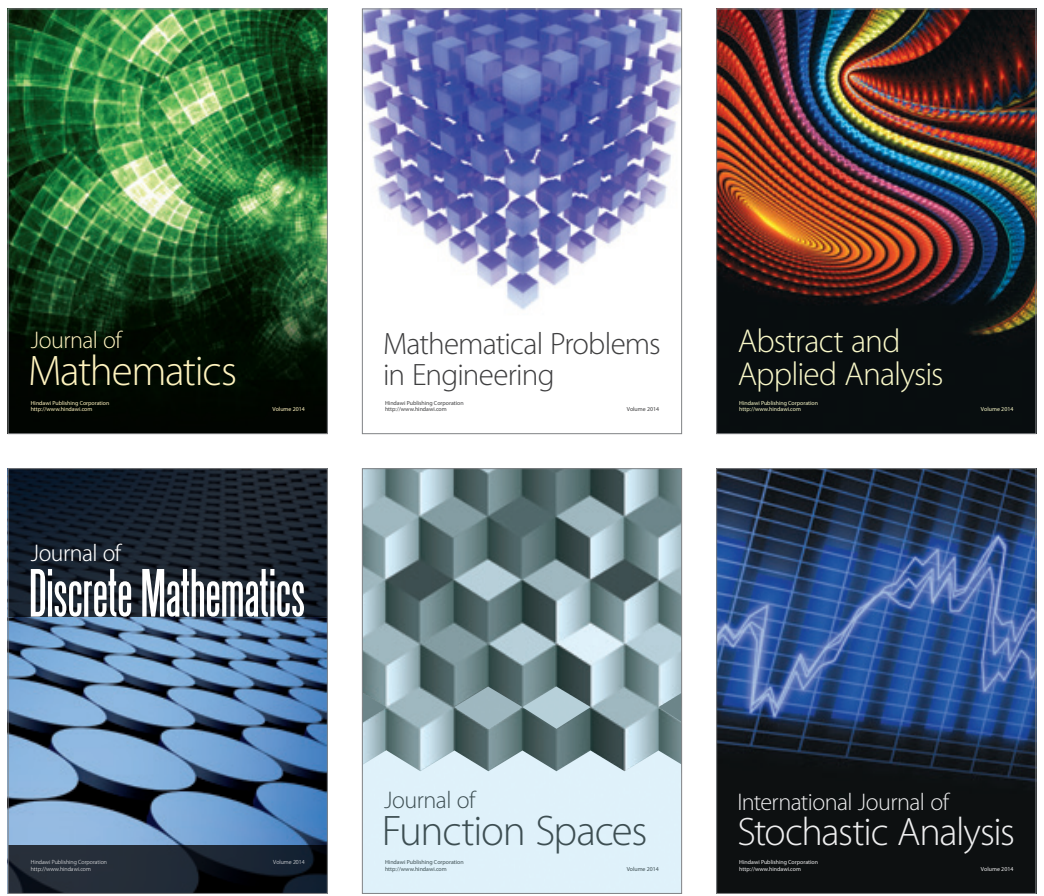

Journal of

Function Spaces

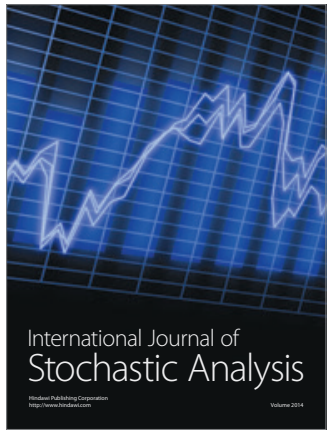

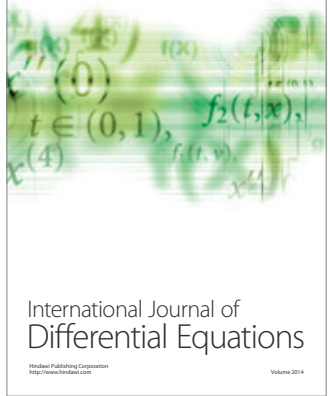
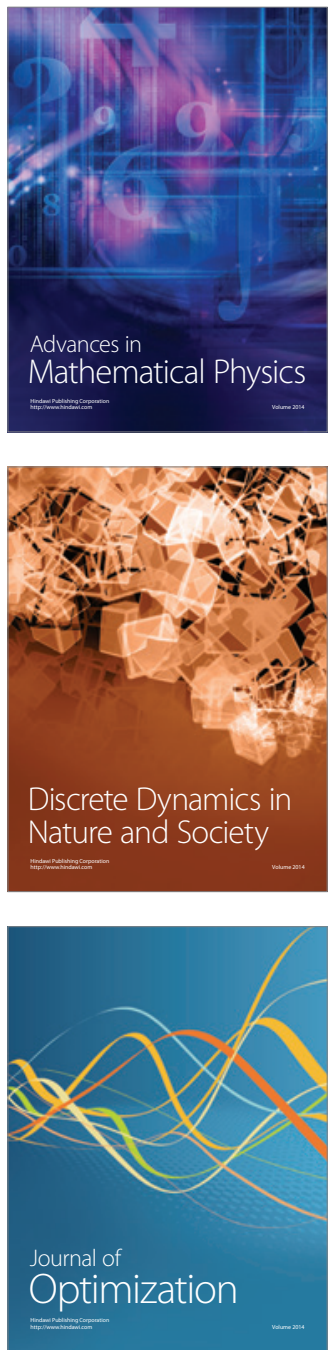